\title{
CONOCIMIENTO E INFERENCIAS EN LA COMPRENSIÓN DE DISCURSOS DE ESPECIALIDAD: EL MANUAL UNIVERSITARIO
}

\author{
Liliana Cubo de Severino \\ Facultad de Filosofía y Letras. Universidad Nacional de Cuyo. \\ Consejo Nacional de Investigaciones Científicas y Tecnológicas \\ lilucubo@speedy.com.ar
}

\begin{abstract}
Resumen
La comprensión de discursos se entiende como un proceso interactivo que requiere por parte del lector la activación de una cantidad considerable de conocimiento previo a fin de generar las inferencias que han sido previstas por el autor del discurso. Pero, dado que es precisamente el conocimiento del que dispone el lector y su relación con lo que lee, el motor que induce a la realización de inferencias ¿qué ocurre cuando existe una relación asimétrica entre autor y lector tal como se da en el caso de los manuales especializados en los que el autor es experto y los lectores suelen tener un grado menor de experticia en la disciplina? A fin de analizar esta interacción, intentaremos responder a dos preguntas de investigación: 1) ¿Qué diferencias cuantitativas y cualitativas existen entre las inferencias que realizan un lector semiexperto y un novato, con diferente conocimiento previo de la disciplina? y 2) ¿Qué inferencias científicas realizan los sujetos de ambos grupos?
\end{abstract}

PALABRAS CLAVE: comprensión de discursos, inferencias, manual especializado, experticia.

\begin{abstract}
Understanding speech is understood as an interactive process. It requires the activation of a considerable amount of prior knowledge to generate inferences that have been provided by the author of the speech. In this work, we analize an asymmetrical relationship between author and reader as given in the case of specialized manuals. In the manuals, the author is an expert and readers generally have a lower degree of expertise in the discipline. In order to analyze this interaction, we will try to answer two research questions: 1) What are the quantitative and qualitative differences between the inferences made by a semiexpert reader and a novice, with different prior knowledge of the discipline? and 2) Which scientific inferences do subjects do in both groups?
\end{abstract}

KEY WORDS: Understanding speech, inferences, specialized manual, expertise.

\section{Introducción}

Las investigaciones sobre comprensión de discursos destacan el complejo proceso inferencial que debe realizar el lector para construir una represen- 
tación mental coherente y al menos parcialmente coincidente con el modelo de discurso construido por el autor (Britton et alii, 1990: 57; Cornish, 1999: 41; Graesser, León y Otero, 2002:12; León, Escudero y van den Broek, 2003:153; Cubo, 2005a: 23; Escudero y León, 2007: 311). En efecto, la comprensión se entiende como un proceso interactivo que requiere, por parte del lector, la activación de una cantidad considerable de conocimiento previo a fin de generar las inferencias que han sido previstas por el autor del discurso.

El tipo de conocimiento previo necesario para la comprensión depende del tipo de texto. Como sabemos, para que el lector pueda realizar inferencias en textos científicos necesita un tipo de conocimiento especializado para reponer la información que falta, a diferencia de lo que ocurre en los textos narrativos, que generalmente requieren información que forma parte de los guiones o planes que todos los que pertenecemos a una misma cultura tenemos en nuestra memoria episódica, por nuestra experiencia de todos los días. Por eso, en los textos narrativos, las inferencias, automáticas o estratégicas, dependen de nuestros Modelos Cognitivos Idealizados, de sentido común o folk (Lakoff, 1987: 68). En el caso de textos de especialidad, en cambio, no solo realizamos inferencias de sentido común sino también inferencias controladas que dependen de un modelo científico previo que el lector debe activar para construir una representación mental coherente (Cubo, 2005b:325; 2009). De allí la importancia que da el autor a los conocimientos científicos presupuestos en el lector potencial.

Pero, dado que es precisamente el conocimiento del que dispone el lector y su relación con lo que lee, el motor que induce a la realización de inferencias ¿qué ocurre cuando existe una relación asimétrica entre autor y lector tal como se da en el caso de los manuales especializados en los que el autor es experto y los lectores suelen tener distintos grados de experticia en la disciplina, según sean expertos, semiexpertos o novatos?

A fin de analizar esta interacción entre lector y autor en la construcción inferencial de la representación mental del modelo de comprensión, abordamos en el presente trabajo la clase textual manual universitario avanzado o especializado, también llamado manual metateórico (Bellert y Weingarten, 1982: 219). Este es un género textual iniciático que constituye una de las fuentes básicas para adquirir los conceptos y métodos de una disciplina. En él, el autor tiene la intención de ayudar al lector semilego (Hyland, 1999: 21; Parodi, 2008: 60) a construir el modelo organizador de un conocimiento nuevo y, en general, explicita los conceptos teóricos necesarios para la comprensión del texto y para controlar el proceso inferencial que realiza el lector. El manual puede ser escrito por un solo autor o construirse como manual 
colectivo en el que distintos autores escriben los diferentes capítulos. En general, en ambos tipos de manuales, los capítulos presentan una parte introductoria, en la que aparece el conocimiento teórico básico que el lector debe conocer para comprender el texto, un núcleo informativo-comunicativo que suele estar en el segmento central del mismo y una parte final que sintetiza los contenidos desarrollados. De acuerdo con estas características funcionales y de estructura del manual, se espera que el lector incorpore los conocimientos nuevos modificando sus esquemas previos, infiera relaciones locales y globales y active e integre información de distintas partes del texto. Esta información nueva debe estar disponible a fin de permitir la realización de inferencias que permitan la comprensión del texto.

Dado que el mayor o menor conocimiento de la disciplina, la mayor o menor experiencia en la lectura de textos especializados y la consiguiente capacidad para incorporar conocimientos nuevos debe influir en la realización de diferentes tipos de inferencias, decidimos emprender una investigación para responder a las siguientes preguntas:

2. ¿Qué diferencias cualitativas y cuantitativas existen entre las inferencias que realizan un lector semiexperto y un novato?

1) ¿Qué inferencias científicas realizan los sujetos de ambos grupos?

Responder a estas preguntas es el objetivo del presente trabajo ${ }^{1}$.

En él presentamos, en primer lugar, el marco de trabajo para el estudio de las inferencias y su clasificación. Luego, en el diseño metodológico, describimos las diferentes técnicas de indagación utilizadas y a continuación presentamos los resultados obtenidos que permiten responder a las dos preguntas de investigación.

\section{Marco Referencial. ¿Qué son las inferencias?}

Como todos sabemos, las inferencias se identifican con representaciones mentales que el lector construye al tratar de comprender un mensaje leído, sustituyendo, añadiendo, eliminando o integrando información del texto (León, 2003: 24).

El mecanismo inferencial funciona a partir de instrucciones tácitas específicas (Cornish, 1999: 5) para operar sobre el modelo mental del discurso que el

1 Una versión parcial del trabajo ha sido presentado en IV Coloquio de Investigadores en Estudios del Discurso y I Jornadas Internacionales de Discurso e Interdisciplina, Universidad Nacional de Córdoba y Regional Argentina de la Asociación Latinoamericana de Estudios del Discurso (ALED), Córdoba, del 16 al 18 de abril de 2009. 
lector va construyendo en colaboración con el autor. Este mecanismo se asienta principalmente en la memoria operativa (Gutiérrez Calvo, 2003: 124) o memoria de corto plazo, que permite realizar diferentes operaciones cognitivas. Mientras el lector procesa la oración focal, la que está leyendo en ese momento, integra información procedente de diferentes fuentes: mantiene en la memoria operativa la representación del texto que está leyendo y activa y recupera de la memoria de largo plazo los conocimientos pertinentes, o bien de la representación del texto previo o bien del conocimiento general de mundo. Simultáneamente, almacena, recupera y procesa. Mantiene de manera accesible a su conciencia de lector los estímulos recién procesados, mientras realiza operaciones cognitivas sobre los estímulos que aparecen a continuación. Estas acciones le permiten relacionar informaciones temporalmente separadas y generar información nueva (Gutiérrez Calvo, 2003:126; Escudero y León, 2007:322). Este proceso es común a la lectura y comprensión de todo tipo de texto y explica, por las limitaciones de la memoria operativa, que no todas las inferencias posibles o potenciales se realicen durante o después de la lectura.

De acuerdo con las características del proceso inferencial descrito, podemos clasificar las inferencias según los procesos de memoria y las fuentes de información utilizada, en tres grandes clases (León, Escudero y van den Broek, 2003:157; Trabasso y Magliano, 1996:256): inferencias hacia atrás, explicativas, que sirven para unir la información de la oración focal con información previa del texto, que forma parte de la representación mental que está construyendo el lector; inferencias concurrentes o asociativas que integran información del conocimiento previo de mundo con la oración focal que se está procesando, e inferencias hacia delante o predictivas, que surgen de expectativas acerca de la continuación del discurso, surgidas de la información contenida en la oración focal y el conocimiento previo.

Estas tres clases de inferencias que, como afirmamos anteriormente, tienen en cuenta las fuentes de información y los procesos de memoria involucrados, a su vez, pueden subdividirse (Cubo de Severino, 2009), siguiendo a Graesser, León y Otero (2002: 10), con modificaciones. Las inferencias explicativas pueden ser anafóricas, puente, causales antecedentes y meta; las inferencias asociativas pueden ser elaborativas y de proceso y las inferencias predictivas, pueden ser causales consecuentes y predictivas no causales, de acuerdo con la siguiente descripción.

1. Las inferencias hacia atrás, de tipo explicativo:

1.1. anafóricas: asignación de un referente a un pronombre o un sintagma nominal que se refiere a un elemento del texto anterior o a una entidad ya introducida en el modelo actual. 
1.2. puente: realización de una inferencia necesaria para relacionar semántica o conceptualmente la oración que se lee con el contenido previo

1.3. causales antecedentes: explicación del suceso o estado que se está leyendo mediante una cadena o una red causal de sucesos y estados anteriores

1.4. meta: atribución del lector a un agente de un motivo que explica una acción intencional a partir de información previa del texto

2. Las inferencias concurrentes con la oración focal o inferencias asociativas:

2.1. atributivas: asociación que realiza el lector, que no se explica mediante mecanismos causales, por la cual infiere propiedades de entidades o hechos.

2.2. de proceso: especificación que realiza el lector de los pasos detallados, las relaciones secuenciales en espacio y tiempo, la forma y las características dinámicas de un suceso mientras se desarrolla.

3. Las Inferencias hacia adelante o inferencias predictivas:

3.1. causales consecuentes: predicción o pronóstico del lector acerca de los sucesos que se deducen causalmente del suceso que está leyendo.

3.2. predictivas no causales: predicción o pronóstico del lector acerca de sucesos futuros que no se deducen causalmente del suceso que está leyendo.

El mecanismo inferencial descrito y la clasificación presentada pueden referirse a la lectura y comprensión de todo tipo de texto. En este sentido, se trata del mismo mecanismo inferencial que realiza tanto el lector de textos expositivos, científicos o no, como el lector de textos narrativos y de otras clases textuales. Las diferencias, si las hay, se referirían a la relación cantidad/ tipo de inferencias según la clase textual y al comportamiento inferencial de distintos tipos de lectores al leer diferentes clases de textos y comprenderlos.

Sin embargo, si consideramos que diferentes tipos de textos exigen la activación de distintos tipos de conocimiento previo podríamos, además, clasificar las inferencias desde el punto de vista de la clase de conocimiento relevante necesario para realizar el proceso (Bellert and Weingartner, 1982: 222; Lakoff, 1987: 33; Seifert, 1993:119) en: 1) inferencias de sentido común o folk, que se basan en información obtenida a partir de la experiencia cotidiana y 2) inferencias científicas que se realizan a partir del conocimiento del modelo científico pertinente.

Estos dos tipos de inferencias corresponden, además, a distintos modos de funcionamiento cognitivo y tipos de causalidad implicados (Escudero y León, 
2007). Las inferencias que surgen del modo científico tienden a establecer relaciones universales con estructura de red causal. Las inferencias de sentido común, en cambio, suelen establecer conexiones, causales o de otro tipo, entre hechos concretos y que no tienden a la generalización.

Las inferencias científicas, por lo tanto, exigen que el lector posea los conocimientos previos necesarios, que se relacionen con el modelo científico que sustenta el autor y sus relaciones de causalidad y que estos conocimientos se activen frente a las claves que aparecen en las oraciones explícitas. De acuerdo con lo anteriormente expuesto, según Britton et al. (1990: 54), las inferencias científicas pueden ser:

- Inferencias externas: son aquellas que requieren conocimiento científico previo que no aparece en el texto, ni en la proposición que se está procesando ni en proposiciones anteriores.

- Inferencias internas: son aquellas que requieren conocimiento científico que aparece en proposiciones anteriores del texto y que exigen que se activen y recuperen los conocimientos pertinentes que están en la representación del texto previo en la memoria de largo plazo y se relacionen con la proposición que se está procesando en la memoria operativa.

\section{Diseño de la investigación}

Material:

En el presente trabajo analizamos las inferencias realizadas a partir de la lectura de subtextos que corresponden a un tipo de secuencia básica (Adam y Revaz, 1996:14): la descripción de proceso, que es una secuencia de uso frecuente en el discurso especializado. Los subtextos pertenecen al capítulo "Métodos experimentales en la investigación del discurso escrito" de Karl Haberland, que forma parte del manual colectivo Conocimiento y discurso (León, 2003: 69) escrito por diferentes autores. El capítulo, tal como su título lo indica, presenta la descripción y evaluación de distintos métodos experimentales que se utilizan en investigaciones psicolingüísticas. Los subtextos seleccionados corresponden a la descripción del proceso que sigue el investigador en cada caso y corresponden al núcleo central del capítulo. En una primera parte del mismo, introductoria, Haberland explicita los conceptos teóricos necesarios para comprender la relación existente entre la teoría y los datos que se pueden obtener con los distintos métodos o técnicas de recolección.

En nuestra investigación utilizamos seis subtextos, normalizados en 300 palabras, en los que se describe respectivamente: métodos de movimientos 
oculares, métodos de ventana, métodos de decisión léxica, métodos de nombrado, métodos de reconocimiento y métodos de preguntas y respuestas. Estos textos tienen una estructura particular que describe la secuencia de acciones que realizan los investigadores con una meta u objetivo y sus relaciones causales, de espacio, de tiempo, de participantes, etc.

Por ejemplo, en el subtexto "Método de la ventana" dice:

En este tipo de método se sigue el siguiente procedimiento: aparece un texto en un terminal de video y el lector va descubriendo segmentos del texto, las llamadas ventanas, mediante la pulsación de una tecla. Los intervalos entre las pulsaciones de la tecla se definen como los tiempos de lectura para la ventana. La ventana puede mostrar el texto completo, frases, oraciones o palabras aisladas [...]" (Haberlandt, 2003: 79).

Y de este modo presenta los distintos pasos y tipos de ventanas que se utilizan y el tipo de información que se obtiene en cada caso.

En el subtexto "Métodos de decisión. El paradigma de priming" dice:

En este paradigma, el sujeto inicialmente lee oraciones y, a continuación, ve pares sucesivos de estímulos de prueba. El primer miembro del par es conocido como prime o inductor, y el segundo, como estímulo crítico o de prueba. Se asume que el prime conecta con su representación en la memoria, activándola y propagando la activación a otros conceptos de la representación [...]" (Haberlandt, 2003: 82).

Y a continuación explica la forma en que los investigadores analizan el patrón de latencias de respuestas a los estímulos.

Sujetos:

La tarea de lectura fue propuesta a dos grupos con diverso nivel de experticia: 24 estudiantes de postgrado de la carrera "Maestría en Ciencias del Lenguaje" y 24 estudiantes de grado de la carrera de Profesorado en Letras que cursaban en sus respectivas carreras la materia Psicolingüística. Se asignó un subtexto a cada sujeto, por lo que cada uno de los seis subtextos fue leído por cuatro sujetos en cada grupo.

Técnicas de indagación:

A fin de responder a las preguntas de investigación, diseñamos dos tareas sucesivas que debían realizar tanto el grupo de sujetos de postgrado como

2 Ambas carreras se dictan en la Facultad de Filosofía y Letras de la Universidad Nacional de Cuyo, Mendoza, Argentina. En las dos carreras me desempeño como profesora titular de Psicolingüística. 
el grupo de sujetos de grado, al leer los subtextos seleccionados. Estas fueron planteadas como tareas de trabajo práctico realizadas en el aula, en instancias de cursado en sus respectivas carreras. Los conocimientos científicos necesarios para comprender estos textos habían sido desarrollados en clase, en semanas anteriores, en ambos grupos, acompañados por la lectura de la primera parte del capítulo en la que el autor desarrolla los conceptos teóricos necesarios para inferir de qué manera podrá interpretar los datos el investigador que aplique las diferentes técnicas descritas.

Las técnicas de indagación utilizadas fueron el protocolo verbal y el test de reconocimiento. La selección de estas herramientas responde a su posibilidad de complementación (León y Escudero, 2003), ya que el protocolo verbal, que es una técnica concurrente, permite interpretar si los sujetos tienen disponible el conocimiento previo necesario para evocar las inferencias potenciales durante la lectura y el test de reconocimiento, que es una técnica retrospectiva (Trabasso y Suh, 1993), permite confirmar si determinadas inferencias potenciales han sido realizadas por el sujeto.

Las técnicas basadas en informes verbales o protocolos son frecuentemente utilizadas para el estudio de las inferencias por su posibilidad de extraer información acerca de: los procesos mentales y el uso de estrategias cognitivas durante la lectura (van Dijk y Kintsch, 1983), de los pensamientos que realizan los alumnos durante la instrucción (Peterson et alii, 1982), de cómo los sujetos se enfrentan a textos que pertenecen a un determinado campo de especialización (Wineberg, 1991) y de las diferencias entre lectores expertos y novatos a la hora de generar distintos tipos de inferencias (Zwaan y Brown, 1996). Si bien la forma convencional del protocolo es oral, existe la posibilidad de aplicar la técnica en forma escrita, ya que se considera a la tarea concepcionalmente oral (Koch-Oesterreicher, 2007) y su implementación por escrito permite eliminar algunos problemas de transcripción.

Muñoz et alii (2006) compararon los resultados obtenidos en protocolos orales y escritos en textos de ciencia y comprobaron que en el caso de lectores expertos, no existen diferencias significativas entre ambas modalidades y en el caso de lectores menos expertos, la modalidad escrita favoreció la formulación de inferencias. Estos resultados explican la selección de la modalidad de protocolo escrito para la presente investigación.

En el caso del test de reconocimiento, el objetivo es medir recuerdo y recuperar inferencias realizadas por el lector y no consignadas en el protocolo. La consigna "reconocer oraciones que están explícitas en el texto" (frente a un listado de oraciones que efectivamente están explícitas, oraciones que actúan como distractores y oraciones que corresponden a posibles inferencias 
realizadas por el lector) se cumple con posterioridad a la lectura, a diferencia del protocolo que se realiza durante la lectura. Influye en esta tarea la naturaleza de la información (Seifert, 1993), de tipo científica, que exige un patrón de activación específico que, puesto en funcionamiento durante el ejercicio de reconocimiento, sirve para conectar y representar relaciones abstractas que aparecen en el texto.

Las tareas propuestas fueron implementadas de la siguiente manera:

1) La primera tarea, realizada independientemente por ambos grupos, consistió en un protocolo verbal (León y Escudero, 2003:100) escrito a posteriori de la lectura de cada oración. El objetivo de lectura planteado fue "estudiar el texto para poder explicar a sus compañeros el proceso que siguen los investigadores en cada caso". Según la consigna de la tarea, los sujetos debían leer cada oración del subtexto asignado y escribir aquellos pensamientos conscientes que llegaban a su mente mientras leían la oración (concurrente en la memoria operativa); podían releer oraciones anteriores y el tiempo era autoadministrado. Se diseñó la experiencia de manera que fuera lo menos intrusiva posible, tanto en lo referido al material de lectura (el texto correspondía a una de las lecturas obligatorias para el cursado de la materia), como a la situación de lectura (leer en clase y tomar notas a medida que se lee un texto científico). El procedimiento permitió conocer los procesos estratégicos conscientes que realizaron los sujetos al leer, sin distinguir entre inferencias de sentido común e inferencias científicas. El posterior conteo y clasificación de las inferencias realizadas orientó en la descripción de la relación cantidad/ calidad de inferencias en este tipo de textos y el comportamiento inferencial de los diferentes grupos según su nivel de experticia. Los resultados permitieron responder la primera pregunta de investigación.

2) A fin de analizar específicamente las inferencias científicas realizadas, aplicamos una segunda tarea, el test de reconocimiento (Seifert, 1993: 109). Se pidió a los 20 sujetos de cada grupo que no habían leído el subtexto "Método de la ventana" que lo leyeran y, luego de un período distractor de 15 minutos, se les presentó un listado de oraciones para que indicaran si las mismas estaban en el texto leído o no. El listado comprendía:

2.1) 10 oraciones que efectivamente aparecen en el texto,

2.2) 10 oraciones que funcionaban como distractores, que no aparecen en el texto ni corresponden a inferencias potenciales $y$,

2.3) 10 oraciones que no aparecen en el texto y corresponden a inferencias científicas internas que debería haber hecho el lector durante la lectura. Estas oraciones pueden considerarse como de conocimiento recientemente adquirido ya que su contenido aparece en la sección introductoria del capítu- 
lo al que pertenece el subtexto leído. En ellas se establecen relaciones científicas entre las técnicas psicolingüísticas y el modelo teórico que el autor ha explicitado en la primera parte del capítulo.

La elección por parte de los estudiantes de las oraciones del tipo 2.3 como pertenecientes al texto, lo que consiste en una falsa alarma, indica que la inferencia fue hecha durante la lectura. El test, aplicado a los dos grupos de sujetos con diferente nivel de conocimiento científico relevante permitió registrar inferencias que habían hecho los lectores y no siempre habían sido explicitadas en el protocolo y mostrar las diferencias de comportamiento inferencial de tipo científico entre ambos grupos, lo que permitió responder a la segunda pregunta de investigación.

\section{Resultados}

4.1. ¿Qué diferencias cualitativas y cuantitativas existen entre las inferencias que realizan un lector semiexperto y un novato, con diferente conocimiento previo de la disciplina y diferente desarrollo de estrategias para leer este tipo de texto?

Los datos obtenidos como resultado de la primera tarea mencionada, el protocolo escrito, mostraron en el grupo de estudiantes de postgrado, un total de 108 inferencias en los seis textos, con una media de 17.92 inferencias por texto. En el grupo de estudiantes de grado, se registró un total de 80 inferencias en los seis textos, con una media de 13.33 inferencias por texto. Esta diferencia muestra, en un primer acercamiento, que la cantidad de inferencias realizadas correlaciona con el nivel de conocimientos y experticia; a mayor experticia mayor número de inferencias, dato que deberá ser corroborado con más datos empíricos.

A fin de analizar la calidad de las inferencias, una vez recogidos los datos -es decir, todas las inferencias que realizaron y explicitaron en el protocolo todos los sujetos- los clasificamos según los procesos de memoria y las fuentes de información utilizada, en las tres grandes clases mencionadas: inferencias hacia atrás o explicativas, inferencias concurrentes con la oración focal que se está procesando o asociativas e inferencias hacia delante o predictivas (León, Escudero y van den Broek, 2003:157; Trabasso y Magliano, 1996:256) y sus subclases.

En la tabla I se exponen los resultados correspondientes a los 24 sujetos del grupo de estudiantes de postgrado. 


\begin{tabular}{|l|c|c|c|c|c|}
\hline \multicolumn{3}{|c|}{ Tipo de Inferencias } & \multicolumn{3}{c|}{ N: 108} \\
\hline Explicativas & total & Asociativas & total & Predictivas & Total \\
\hline Anafórica & $9 \%$ & Elaborativa & $7 \%$ & Causal consecuente & $16 \%$ \\
\hline Puente & $6 \%$ & De proceso & $10 \%$ & Predictiva no causal & $12 \%$ \\
\hline Causal antecedente & $33 \%$ & & & & \\
\hline Meta & $7 \%$ & & & & \\
\hline TOTAL & $55 \%$ & TOTAL & $17 \%$ & TOTAL & $28 \%$ \\
\hline
\end{tabular}

Tabla I. Porcentaje de inferencias explicitadas por estudiantes de postgrado

Según se observa en la tabla I, las inferencias explicativas hacia atrás son las más frecuentes, con un 55\% y entre ellas las causales antecedentes que representan un 33\% del total. En orden de frecuencia siguen las inferencias predictivas hacia adelante, con un $28 \%$ y entre ellas las causales consecuentes con un $16 \%$. Estos datos confirman la tendencia observada por distintos investigadores (Escudero y León, 2007: 315) referida a los patrones de procesamiento del discurso científico en general, es decir, que este tipo de textos tiene la relación causal como principio organizativo básico. Llama la atención el bajo porcentaje de inferencias explicativas de meta, ya que por hipótesis esperábamos que los lectores relacionaran los métodos experimentales con las acciones intencionadas del investigador. La clase de inferencias asociativas concurrentes, la menos frecuente, representó solo un 17\% del total, con el mayor porcentaje en inferencias de proceso, tal como era esperable.

La misma tarea realizada por alumnos de grado mostró los siguientes porcentajes:

\begin{tabular}{|c|c|c|c|c|c|}
\hline \multicolumn{4}{|c|}{ Tipo de Inferencias } & \multicolumn{2}{|l|}{$\mathrm{N}: 80$} \\
\hline Explicativas & total & Asociativas & total & Predictivas & Total \\
\hline Anafórica & $15 \%$ & Elaborativa & $20 \%$ & Causal consecuente & $15 \%$ \\
\hline Puente & $2 \%$ & De proceso & $15 \%$ & Predictiva no causal & $5 \%$ \\
\hline Causal antecedente & $26 \%$ & & & & \\
\hline Meta & $2 \%$ & & & & \\
\hline TOTAL & $45 \%$ & TOTAL & $35 \%$ & TOTAL & $20 \%$ \\
\hline
\end{tabular}

Tabla II. Porcentaje de inferencias explicitadas por estudiantes de grado

Según se observa en la Tabla II, la tendencia general, aún en lectores con menos experiencia y conocimiento, es de una mayor frecuencia de inferencias 
explicativas, si bien en menor medida que los estudiantes de posgrado ( $55 \%$ / 45\%) y entre ellas, también las causales antecedentes son las más frecuentes. Las inferencias predictivas causales consecuentes presentan una frecuencia similar en ambos grupos. Esto nos permite confirmar, al menos a la luz de estos datos empíricos, que en la lectura de la clase textual manual los lectores con diferente nivel de experticia intentan generar una explicación y predicción de tipo causal.

Es interesante observar la diferencia en el comportamiento de ambos grupos en relación con las inferencias anafóricas, ya que los lectores expertos, con mayor experiencia en la lectura de este tipo de texto, no siempre explicitaron inferencias que necesariamente deben haber realizado, probablemente de manera automática, como las anafóricas, a diferencia de los menos expertos que analizaron las anáforas de modo más consciente y consignaron esta tarea.

Por otra parte, al igual que en los estudiantes de postgrado, en el grupo de novatos, las inferencias de meta presentan baja frecuencia, dato no esperado que deberá ser estudiado en futuras investigaciones y que, en principio, creemos que puede deberse al tipo de procesamiento local, oración por oración, que exigía la tarea solicitada.

Una diferencia importante entre los dos grupos se observa en relación con las inferencias asociativas, que son más frecuentes en el grupo con menor conocimiento y experticia y se refieren a la dirección en la que el lector busca o conecta la información. En efecto, la mayor frecuencia de inferencias asociativas revela la tendencia a comprender el significado literal y local del texto más que a integrar las distintas oraciones de manera global. Esto podría revelar una incapacidad para activar en memoria, simultáneamente, información nueva recientemente adquirida y mostraría el tipo de información que está accesible en la memoria de trabajo. Esta información está referida a la oración focal o concurrente que en ese momento se procesa y que en el caso de los subtextos leídos se refiere a quién hace qué, con qué, cuándo y dónde, información que se basa más en el conocimiento general de mundo del lector que en el conocimiento teórico aparecido previamente en el texto.

De acuerdo con los datos, que muestran las inferencias realizadas conscientemente y explicitadas en el protocolo y más allá de las diferencias observadas en el comportamiento inferencial de ambos grupos, podemos concluir que la clase de texto, el manual especializado en lingüística, en razón de que su estructura y contenido presenta la relación causal como principio organizativo básico, promueve en el lector la realización de un patrón de inferencias en el que predominan las relaciones causales. 


\section{2. ¿Qué inferencias científicas realizan los sujetos de ambos grupos?}

A fin de investigar si los sujetos habían realizado algunas inferencias científicas internas necesarias para comprender el texto, aplicamos un test de reconocimiento (Seifert, 1993: 114). Tal como expusimos previamente, pedimos a 20 estudiantes de cada grupo, que no habían leído previamente el texto del "Método de la ventana", que lo leyeran, autoadministrando su tiempo de lectura y luego de un período distractor de 15 minutos, les presentamos un listado de oraciones a fin de que marcaran las que sí estaban en el texto.

Las oraciones presentadas, que aparecían intercaladas, correspondían a tres clases:

1) 10 oraciones que efectivamente aparecen en el texto,

2) 10 oraciones que funcionan como distractores y que no están en el texto,

3) 10 oraciones que no están en el texto y que corresponden a inferencias científicas internas que debería haber hecho el lector durante la lectura para construir la coherencia.

Si los sujetos marcaban las oraciones tipo 3 como explícitas en el texto, esto indicaba que habían hecho la inferencia correspondiente durante o después de la lectura. Este reconocimiento se considera falsa alarma.

Los resultados obtenidos en la muestra de los dos grupos de 20 sujetos, en relación con el marcado de las oraciones tipo 3 aparecen en la siguiente tabla:

\begin{tabular}{|c|c|c|c|}
\hline \multicolumn{4}{|c|}{ Falsa alarma } \\
\hline \multicolumn{2}{|c|}{ Alumnos de postgrado } & \multicolumn{2}{c|}{ Alumnos de grado } \\
\hline $\begin{array}{c}\text { Cantidad de oraciones tipo } 3 \\
\text { reconocidas por los } 20 \\
\text { alumnos }\end{array}$ & Promedio & $\begin{array}{c}\text { Cantidad de oraciones tipo } 3 \\
\text { reconocidas por los 20 } \\
\text { alumnos }\end{array}$ & Promedio \\
\hline $\begin{array}{c}7,8,6,9,7,8,7,8,5,7, \\
7,7,8,9,6,8,8,7,6,9\end{array}$ & 7.35 & $\begin{array}{c}5,6,4,5,3,6,5,7,7,4, \\
4,5,5,5,3,6,6,5,5,5\end{array}$ & 5,05 \\
\hline
\end{tabular}

Tabla III. Porcentaje de oraciones correspondientes a inferencias científicas

De acuerdo con los datos, sobre un total de 10 inferencias científicas internas necesarias para la comprensión del texto, los estudiantes de postgrado obtuvieron una media de 7.35 y los estudiantes de grado, una media de 5.05, lo que muestra como tendencia general, tal como esperábamos, una mayor capacidad para realizar inferencias científicas en los sujetos con mayor experticia. Sin embargo, tal como se muestra en la tabla, existe una variación individual importante que queda reflejada en los datos que decidimos colocar en bruto para su mejor visualización. 
Las diferencias observadas entre los dos grupos muestra la influencia del conocimiento previo recién adquirido sobre un dominio específico y la capacidad para activarlo rápidamente e interactuar con la información entrante durante la lectura. Esto habla de los distintos grados de experticia o pericia de los dos grupos de sujetos en ese dominio.

Esta diferencia permite reconocer, en los estudiantes de postgrado, mayor accesibilidad a los conocimientos científicos recién adquiridos, o sea, mayor eficiencia en los sujetos para encontrar una información en la memoria y relacionarla con la información que se está procesando (Gutiérrez Calvo, 2003: 137).

\section{Discusión Final}

Tal como expusimos en la introducción, el objetivo de la presente investigación es analizar la interacción entre autor y lectores con diferente nivel de experticia, en la construcción inferencial de la representación mental del modelo de comprensión del manual universitario especializado. Los resultados obtenidos en relación con la actividad inferencial se derivan, en parte, de las características del material de lectura presentado y, en parte, del uso de estrategias específicas que surgen como consecuencia de la aplicación de las técnicas de indagación utilizadas.

Teniendo en cuenta estas limitaciones, podemos hacer algunas generalizaciones como respuesta a las preguntas de investigación planteadas: ¿qué diferencias cualitativas y cuantitativas existen entre las inferencias que realizan un lector semiexperto y un novato, con diferente conocimiento previo de la disciplina y diferente desarrollo de estrategias para leer este tipo de texto? y ¿qué inferencias científicas realizan los sujetos de ambos grupos?

Si observamos desde el paradigma experto-novato las diferencias que surgen de los datos al comparar los procesos que utilizan y cómo organizan y recuperan el conocimiento ambos grupos, advertimos que el grupo de sujetos con menor grado de experticia centra su atención en el procesamiento de cada oración de manera independiente y, en general, es poco eficiente en la recuperación de información que se relaciona con la representación mental global del texto. Esto se ve reflejado en el comportamiento de los novatos, por una parte, en el mayor número de inferencias asociativas y menor número de inferencias explicativas comparados con los lectores semiexpertos, lo que muestra la dirección en la que el lector busca o conecta la información. Por otra parte, se puede observar en el bajo rendimiento obtenido por estos sujetos en el test de reconocimiento de oraciones que corresponden a inferencias científicas internas, que recuperan conocimiento científico recién adquirido. 
En efecto, los alumnos de grado establecieron, en muchos casos, relaciones causales a partir de su conocimiento general de mundo o conocimiento cotidiano, referidas a los pasos y la dinámica seguida en la aplicación de cada técnica expuesta en el subtexto leído, pero no se preguntaron por qué realizaba el investigador esa tarea ni qué generalización se podía realizar con la aplicación de la misma. Si bien habían estado en contacto con el conocimiento científico necesario para establecer relaciones causales científicas, al leer la introducción, no siempre activaron este conocimiento o bien porque no lo reconocieron como pertinente o bien porque no había sido internalizado previamente.

Los lectores más expertos, en cambio, establecieron más relaciones causales a partir de conocimiento científico pertinente y recuperaron supuestos teóricos básicos que estaban en la primera parte del capítulo, lo que les permitió comprender no solo la descripción del procedimiento que se explicaba en el texto, sino también interpretar la lógica general de la experimentación planteada.

Por ejemplo, frente a la oración:

"[...] De esta manera las palabras parecen marchar por la pantalla a un ritmo impuesto por las presiones de las teclas por parte del lector [...]" un sujeto semiexperto consigna la siguiente inferencia científica interna "[...] si el lector demora más en leer la palabra quiere decir que esta palabra tiene más carga y exige un procesamiento más largo [...]".

Frente a la misma oración, un sujeto no experto consigna la siguiente inferencia asociativa de proceso: "[...] El lector aprieta la tecla luego de haber leído la palabra [...]".

Este ejemplo muestra las diferencias en el tipo de funcionamiento cognitivo y las diferentes formas de establecer relaciones, en el caso del semiexperto de tipo causal, con generalizaciones y en el caso del novato de tipo temporal, referida al proceso serial en un hecho concreto. Inferencia científica en el primer caso e inferencia de sentido común en el último.

El análisis de la interacción permite concluir que la comprensión de manuales especializados requiere por parte del lector la activación de conocimiento científico previo que debe haber sido internalizado a fin de que se active y permita generar las inferencias que han sido previstas por el autor del discurso, por lo que es necesario un nivel de experticia para la representación global del texto que no siempre posee el novato.

Esto exige, en el caso de las tareas propuestas, que el lector haya incorporado a sus esquemas previos los conocimientos nuevos explicitados por el autor en la primera parte del capítulo, que los active y los relacione causalmente con el procedimiento experimental explicado en el subtexto. Este proceso inferencial no fue totalmente exitoso en los alumnos de grado, creemos, 
debido a que estos no han logrado incorporar los nuevos conocimientos, no han logrado activarlos o no los han relacionado con las oraciones leídas. Dada la importancia que tiene este tipo de texto en la formación científica de los estudiantes, creemos necesaria la realización de futuros trabajos con datos empíricos a fin de determinar cuáles de estas estrategias han desarrollado los lectores y cuáles no.

\section{Referencias bibliográficas}

Adam, J. M. y F. Revaz (1996): "(Proto) tipos: La estructura de la composición en los textos", Textos de Didáctica de la lengua y la literatura, 10, págs. 9-22.

Bellert, I. y P. Weingartner (1982): "On different characteristics of scientific texts as compared with everyday language texts", en Kittredge R. y J. Lehrberger (eds.): Sublanguage. Studies of Language in Restricted Semantic Domains. NY: Walter de Gruyter, págs. 219-231.

Britton, B., Van Dusen, L., Glynn, S. y Hemphill, D. (1990): "The impact of inferences on instructional text", en Graesser, A. y G. Bower (eds.): Inferences and Text Comprehension. San Diego, CA, Academic Press, págs. 53-70.

Cornish, F. (1999): Anaphora, Discourse, and Understanding. Evidence from English and French. New York, Oxford University Press.

Cubo de Severino, L. (2002): "Evaluación de estrategias retóricas en la comprensión de manuales universitarios", Revista del Instituto de Investigaciones Lingüísticas y Literarias Hispanoamericanas, (RILL), XV, págs. 55- 59.

Cubo de Severino, L. (ed.) (2005a): Leo, pero no comprendo. Córdoba (Argentina), Comunicarte.

Cubo de Severino, L. (2005b): "Los manuales universitarios". En Cubo de Severino, L. (ed.): Los textos de la ciencia. Principales clases de discurso Académico-Científico. Córdoba (Argentina), Comunicarte, págs. 325- 336.

Cubo de Severino, L. (2009): "Discurso académico científico y perfil inferencial: el manual especializado", IV Coloquio de Investigadores en Estudios del Discurso y I Jornadas Internacionales de Discurso e Interdisciplina. Córdoba (Argentina), Universidad Nacional de Córdoba.

Escudero, I. y J. León (2007): "Procesos inferenciales en la comprensión del discurso escrito. Influencia de la estructura del texto en los procesos de comprensión", Signos 40 (64), págs. 311-336.

Graesser, A., J. León y J. Otero (2002): "Introduction to the psychology of science text comprehension", en Otero, J., León, J. y Graesser, A. (eds.): The psychology of science text comprehension. Mahwah, NJ, Lawrence Erlbaum Associates, págs. 1-15.

Gutiérrez Calvo, M. (2003): "Memoria operativa e inferencias en la comprensión del discurso”. En León, J. (ed.): Conocimiento y discurso. Madrid, Pirámide, págs. 123-138.

Haberlandt, K. (2003): "Métodos experimentales en la investigación del discurso escrito”. En León, J. (ed.). Conocimiento y discurso, Madrid, Pirámide, págs. 69-96. 
Hyland, K. (1999): "Talking to Students: Metadiscourse in introductory coursebooks", English for Specific Purposes, 18 (1), págs. 3-26.

Koch, P. y Oesterreicher, W. (2007): Lengua hablada en la Romania: español, francés, italiano. Madrid, Gredos.

Lakoff, G. (1987): Women, Fire, and Dangerous Things. What Categories Reveal about the Mind. Chicago, University of Chicago Press.

León, J. A. (ed). (2003): Conocimiento y discurso. Madrid, Pirámide.

León, J. e I. Escudero (2003): "Protocolos verbales en el estudio de las inferencias: una metodología emergente". En León, J. (ed.): págs. 99-119.

León, J., Escudero, I. y Van den Broek, P. (2003): "La influencia del género del texto en el establecimiento de inferencias elaborativas”. En León, J. (ed.), págs. 153-169.

Muñoz, B., Magliano, J., Robin Sheridan, R. y McNamara, D. (2006): “Typing versus thinking aloud when reading: Implications for computer-based assessment and training tools", Behavior Research Methods, 38 (2), págs. 211-217.

Parodi, G. (ed.) (2008): Géneros Académicos y Géneros Profesionales: Accesos Discursivos para Saber y Hacer. Valparaíso, Ediciones universitarias de Valparaíso.

Peterson, P., Swing, S., Braverman, M. y Buss, R. (1982): "Students' aptitudes and their reports of cognitive processes during instruction", Journal of Educational Psychology, 74, págs. 535-547.

Seifert, C. (1993): "Content-based inferences in text". En Graesser, A. y Bower, G. (eds.): Inferences and Text Comprehension. San Diego, CA, Academic Press, págs. 103-121.

Trabasso, T. y Magliano, J. (1996): "Conscious understanding during text comprehension”, Discourse Processes, 21, págs. 255- 287.

Trabasso, T. y Suh, S. (1993): "Understanding text: Achieving explanatory coherente through on-line inferences and mental operations in working memory", Discourse Processes, 16, págs. 3-34.

Van Dijk, T. y Kinstch, W. (1983): Strategies of the discourse comprehension. New York, London Academic Press.

Wineberg, S. (1991): "On the reading of historical texts: Notes on the breach between school and academy", American Educational Research Journal, 28, págs. 495-520.

Zwaan, R. y Brown, C. (1996): "The influence of language proficiency and comprensión skill on situation-model construction”, Discourse Processes, 21, págs. 289-327. 